\title{
Reinfecção por COVID-19: Uma revisão das novas evidências
}

\section{COVID-19 Reinfection: A review of the new evidence \\ Reinfección de COVID-19: Una revisión de la nueva evidencia}

Wellington Manoel da Silva ORCID: https://orcid.org/0000-0002-6735-5071 Instituto de Medicina Integral Professor Fernando Figueira, Brasil E-mail: wellington-manoel@outlook.com

Maria Eduarda da Silva ORCID: https://orcid.org/0000-0003-3070-4992 Universidade Federal de Pernambuco, Brasil E-mail: me89075@gmail.com

Jociéllen Maria Gomes de Lima ORCID: https://orcid.org/0000-0002-4893-4617 Universidade Federal de Pernambuco, Brasil E-mail: jociellen07@ hotmail.com

Analice Pereira Canejo Ferreira ORCID: https://orcid.org/0000-0002-0728-7433 Universidade de Pernambuco, Brasil E-mail: analicepcf@gmail.com

Francisca Vanessa de Oliveira ORCID: https://orcid.org/0000-0003-4193-7855 Faculdade Santa Maria, Brasil E-mail: fvanessaoliv@gmail.com

Kerolain Alves Federici de Souza

ORCID: https://orcid.org/0000-0001-9136-8529 Faculdade de Comunicação Tecnologia e Turismo de Olinda, Brasil E-mail: kerolainfalves@gmail.com

Juliana Figueira de Carvalho Pereira ORCID: https://orcid.org/0000-0003-4308-8551 Faculdade de comunicação e turismo de Olinda, Brasil E-mail: juliana_fcp@hotmail.com

Jardeson Joaquim Bezerra

ORCID: https://orcid.org/0000-0003-4449-4199 Universidade Federal do Rio Grande do Norte, Brasil

E-mail: jardesonbezerra1990@gmail.com

Marks Passos Santos

ORCID: https://orcid.org/0000-0003-1180-404X Faculdade Ages de Medicina, Brasil E-mail: marks.santos@ ages.edu.br

Williane Karine Lira Barros da Silva ORCID: https://orcid.org/0000-0003-4289-966X Universidade Federal da Paraíba, Brasil E-mail: willianekbarros@gmail.com

Joyce Neire Vidal Alexandre Souza ORCID: https://orcid.org/0000-0003-2083-4566 Universidade de Pernambuco, Brasil E-mail: joyceneire@hotmail.com

Thayná Karollyne Carvalho Silva ORCID: https://orcid.org/0000-0002-4848-1208

Universidade Federal de Pernambuco, Brasil E-mail: thayna_karollyne@hotmail.com

Edilany Keline de Oliveira Silva

ORCID: https://orcid.org/0000-0002-3929-5091 Faculdade Integração do Sertão, Brasil E-mail: enfedilanykeline@ hotmail.com

Cassandra Alves de Oliveira Silva ORCID: https://orcid.org/0000-0003-3478-8177 Universidade Federal da Paraíba, Brasil E-mail: cassandra.aiesec@gmail.com

Jamille Maria Moreira da Silva ORCID: https://orcid.org/0000-0001-6074-945X União de Ensino Superior de Campina Grande, Brasil E-mail: jamille.moreira@hotmail.com 


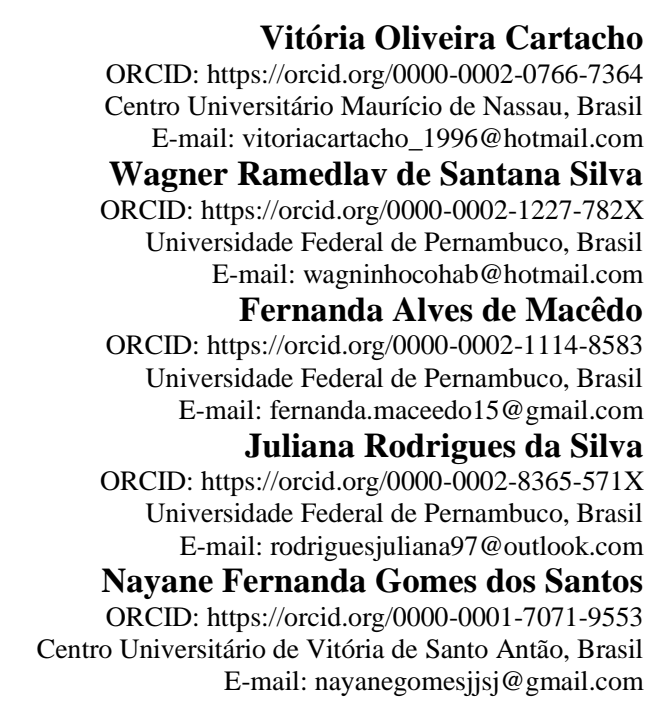

Resumo

O surto da doença provocada pelo novo coronavírus em 2019 (COVID-19) começou em dezembro de 2019 e rapidamente espalhou-se pelo mundo como uma grande ameaça à saúde. Diversos relatos de casos positivos após alta hospitalar têm chamado a atenção de pesquisadores ao redor do mundo. Desta forma, o presente estudo busca relatar os principais resultados das mais recentes evidências científicas a respeito da temática. Para tanto, foi realizada uma revisão narrativa utilizando as principais bases de dados contidas na Biblioteca Virtual em Saúde, a saber, Medical Literature Analysis and Retrieval System Online - MEDLINE e Literatura Latino-Americana e do Caribe em Ciências da Saúde - LILACS. A curta duração da soropositividade para anticorpos neutralizantes, a presença de mais de variantes genotípicas do vírus, além de os níveis de imunoglobulina poderem não se correlacionar com a eliminação viral e o risco de transmissibilidade de SARS-CoV-2 aumentam os riscos de reinfecção pelo vírus, tornando extremamente necessária a disseminação de medidas de prevenção a infecção, até que mais aspectos do vírus sejam conhecidos e que haja uma vacina eficaz e capaz de promover a imunidade coletiva.

Palavras-chave: Infecções por coronavírus; Pandemias; Imunidade coletiva; Covid-19.

\begin{abstract}
The outbreak of the disease caused by the new coronavirus in 2019 (COVID-19) began in December 2019 and quickly spread across the world as a major health threat. Several reports of positive cases after hospital discharge have drawn research attention around the world. Thus, the present study seeks to report the main results of the most evidences scientific evidence regarding the theme. To this end, a narrative review was carried out using the main databases contained in the Virtual Health Library, a saber, Medical Literature Analysis and Retrieval System Online MEDLINE and Latin American and Caribbean Literature in Health Sciences - LILACS. The short duration of seropositivity for neutralizing antibodies, the presence of more than genotypic variants of the virus, in addition to the fact that immunoglobulin levels may not correlate with viral elimination and the risk of SARS-CoV-2 transmissibility increase the risk of reinfection by viruses, making it extremely necessary to disseminate measures to prevent infection, until more aspects of the virus are known and there is an effective vaccine capable of promoting colletive immunity.
\end{abstract}

Keywords: Coronavirus infections; Pandemics; Collective immunity; Covid-19.

\title{
Resumen
}

El brote de la enfermedad causada por el nuevo coronavirus en 2019 (COVID-19) comenzó en diciembre de 2019 y se extendió rápidamente por todo el mundo como una importante amenaza para la salud. Varios informes de casos positivos después del alta hospitalaria han atraído la atención de investigadores de todo el mundo. Así, el presente estudio busca reportar los principales resultados de la evidencia científica más reciente sobre el tema. Para ello, se realizó una revisión narrativa utilizando las principales bases de datos contenidas en la Biblioteca Virtual en Salud, a saber, Sistema de Análisis y Recuperación de Literatura Médica en Línea - MEDLINE y Literatura Latinoamericana y Caribeña en Ciencias de la Salud - LILACS. La corta duración de la seropositividad para los anticuerpos neutralizantes, la presencia de más variantes genotípicas del virus, además del hecho de que los niveles de inmunoglobulina pueden no correlacionarse con la eliminación viral y el riesgo de transmisibilidad del SARS-CoV-2 aumentan el riesgo de reinfección por virus, por lo que es sumamente necesario difundir medidas para prevenir la infección, hasta que se conozcan más aspectos del virus y exista una vacuna eficaz capaz de promover la inmunidad colectiva.

Palabras clave: Infecciones por coronavirus; Pandemias; Inmunidad colectiva; Covid-19. 


\section{Introdução}

Diversos fatores contribuíram para a rápida evolução da doença do novo coronavírus (COVID-19) de uma doença endêmica para uma pandemia (Hanaei \& Rezaei, 2020). Alguns podem ser atribuídos as características do vírus da síndrome respiratória aguda grave (SARS-CoV-2), como alto número de reprodução básica (R0) de cerca de 2 a 6 e transmissibilidade, apesar da ausência de clínica manifestações por vezes (Randolph \& Barriero, 2020).

No entanto, muitas suposições sobre a pandemia, a maioria das quais foram falsificadas ou questionadas, neste ponto, também desempenharam um papel importante. Um tal pressuposto era que SARS-CoV-2, semelhante ao vírus H1N1, iria atenuar em altas temperaturas, enquanto que na verdade observa-se a segunda onda da pandemia em alguns países, apesar do aumento das temperaturas. Outra suposição era alcançar imunidade de rebanho para SARS-CoV-2 com aumento do número de indivíduos infectados, restringindo assim a transmissão do vírus devido ao aumento do número de hospedeiros imunes e diminuição do número de suscetíveis (Lofti \& Rezaei, 2020).

No entanto, um conceito-chave no estabelecimento e manutenção da imunidade de rebanho é uma forte imunização contra os patógenos duradoura o suficiente para que a fração da população imune ao vírus ultrapasse a imunidade de rebanho limite. Portanto, a persistência das concentrações de imunoglobulina são um dos fatores determinantes na possibilidade de alcançar imunidade coletiva (Randolfi \& Barriero, 2020).

$\mathrm{Na}$ maioria dos indivíduos infectados com SARSCoV-2, os níveis de imunoglobulina neutralizante ( $\operatorname{IgM}$ e $\operatorname{IgG})$ aumentam dentro de dias a semanas após o início dos sintomas (Lofti \& Rezaei, 2020). Esses anticorpos são responsáveis por produzir imunidade à reinfecção em primatas desafiados novamente com SARS-CoV-2 28 dias após a infecção inicial. Contudo, ao contrário de muitos outros vírus respiratórios, resultando em concentrações de imunoglobulinas que duram vários meses, as imunoglobulinas neutralizantes contra SARS-CoV-2 persistem por cerca de 40 dias apenas (Kirkcaldy king \& Brooks, 2020).

Por outro lado, testes de RNA positivos, apesar da soropositividade para IgG após infecção primária, vêm sendo amplamente relatados (Roy, 2020). Mesmo que tais casos possam ser interpretados como portadores silenciosos ou relacionados à baixa confiabilidade para os kits disponíveis comercialmente e/ou erros de amostragem, a janela de tempo entre a principal infecção e o segundo teste de RNA positivo, que é cerca de 2 meses, em alguns casos, pode ser sugestivo de reinfecção com o vírus ou reatividade de uma infecção latente com SARS-CoV-2 (Roy, 2020).

Recentemente foi confirmado no Brasil o primeiro caso de reinfecção por COVID-19, no dia 9 de dezembro de 2020 (Fiocruz, 2020), desta forma, faz-se necessário analisar o que as experiências internacionais sobre o tema têm relatado, afim de, possibilitar uma melhor análise e conduta para o enfrentamento desta problemática. Assim o objetivo deste estudo é relatar casos de reinfecção por SARS-CoV-2 evidenciados na literatura internacional e discutir a problemática à luz da imunopatologia.

\section{Metodologia}

Este estudo trata-se de um estudo de revisão de literatura, do tipo narrativa. Entende-se este tipo de revisão como uma análise bibliográfica mais ampla, não sendo necessário determinar-se uma metodologia rigorosa que possibilite a reprodução da pesquisa (Vosgerau \& Romanowsk, 2014).

Entretanto, é um método importante para a obtenção de um panorama geral do conhecimento acerca de um determinado tema, capaz de contribuir com a apresentação de novas evidências (Elias et al., 2012).

A busca da literatura aconteceu de novembro a dezembro de 2020, sendo encontrados inicialmente 149 artigos, utilizando a plataforma Biblioteca Virtual em Saúde, com os descritores: Infecções por coronavírus e recidiva utilizando o operador booleano "AND” para a associação entre estes. O levantamento foi realizado considerando as bases de dados: 
Literatura Latino Americana e do Caribe em ciências da Saúde (LILACS) e Medical Literature Analysis and Retrieval System Online (MEDLINE).

Para a seleção dos estudos, utilizou-se os seguintes critérios de inclusão: artigos originais, publicados no período de março a dezembro 2020, no idioma inglês. Os critérios de exclusão foram: artigos duplicados, indisponíveis na íntegra e que não abordassem a temática. Assim, ao final, foram selecionados 18 artigos para análise e discussão.

\section{Resultados e Discussão}

\subsection{A pandemia da COVID-19}

O surto da doença provocada pelo novo coronavírus (COVID-19) teve seu início em dezembro de 2019, em Wuhan, província de Hubei, China, espalhou-se pelo mundo tornando-se um grande problema de saúde sem precedentes (W. M. Silva et al., 2020). A partir de 3 de julho de 2020, COVID- 19 foi responsável por mais de 12 milhões de casos confirmados, incluindo mais de 570 mil mortes em 216 países, e o número de casos ainda está em crescente aumento (World Health Organization, 2020).

Os sintomas da COVID-19 podem incluir febre, tosse, falta de ar, dor de cabeça, dor de garganta, fadiga, perda do paladar (afagia ou disfagia) ou do cheiro (anosmia), náuseas, vômitos e sintomas intestinais como diarreia (Centers of Disease Control, 2020). A grande maioria dos casos de COVID-19 são leves, enquanto alguns indivíduos (14\%) desenvolvem formas graves da doença, necessitando de oxigenoterapia, e cerca de 5\% precisam ser internados em unidade de terapia intensiva (WHO, 2020).

Foram relatados em casos graves de COVID-19, complicações como síndrome do desconforto respiratório agudo, sepse, choque séptico e falência de múltiplos órgãos (Yang et al., 2020; M. E. Silva et al., 2020). Os indivíduos acometidos por formas leves da COVID-19 são geralmente internados no hospital para receber tratamento padrão e, adquirindo melhora clínica, recebem alta de acordo com os protocolos e diretrizes emitidos pelas autoridades de saúde locais.

Geralmente, os pacientes têm recebido alta quando estão, há pelo menos, 3 dias sem febre e após pelo menos tendo obtido dois resultados negativos consecutivos do teste de reação de transcriptase reversa em tempo real (RT-qPCR) e não possuindo nenhum sintoma no momento da alta hospitalar (European Centre for Disease Prevention and Control, 2020).

\subsection{A reinfecção pelo SARS-CoV-2: o que as evidências nos mostram?}

O fenômeno de PCR re-positivo para COVID-19 foi amplamente relatado configurando-se como mais um desafio emergente do controle global da pandemia. Uma das séries de casos de COVID-19 re-positivo de maior impacto foi relatada pelos Centros de Controle e Prevenção de Doenças da Coreia (KCDC), nos quais eles conduziram uma extensa investigação epidemiológica envolvendo 285 casos re-positivos e 790 contatos.

Durante sua triagem de rotina em pacientes assintomáticos, foi relatada, ainda, uma alta detecção de casos re-positivos de 44,7\% (126 de 284) entre os pacientes assintomáticos (Korea Centres for Disease Control and Prevention, 2020). Zhang et al. em Guangdong, China, investigaram as características clínicas e laboratoriais de sete pacientes que foram readmitidos devido a ensaios de PCR re-positivos.

Os autores relataram que durante o período de isolamento no hospital, quatro resultados foram positivos apenas para esfregaços retais, dois foram positivos para esfregaços de garganta e um paciente teve esfregaços retais e de garganta positivos (Zhang et al., 2020).

Outro estudo, realizado por $\mathrm{Li}$ et al. em Chongqing, China concentrou-se na identificação de 19 pacientes que tiveram resultados positivos de RT-qPCR após receberem alta (Li, et al., 2020). Luo et al. de Wuhan, China, relataram o caso de uma mulher de 58 anos com resultados flutuantes persistentes para o teste COVID-19 (Luo, 2020). 
Outro relatório sobre resultados flutuantes foi relatado em um estudo de Xing et al. em Wuhan, China, envolvendo dois casos (Xing et al., 2020). Em Chongqing, China, Chen et al. relataram os resultados de outros quatro pacientes, três dos quais tiveram resultados positivos para swabs nasofaríngeos, e um teve resultado positivo para swab anal, após 3 dias de ter recebido alta (Chen et al., 2020b).

Em Shenzhen, China, um estudo observou que 20 de 182 pacientes assintomáticos (10,99\%) obtiveram testes positivos após resultados negativos iniciais para RNA do SARS-CoV-2, causador da síndrome respiratória aguda grave (Yuan et al., 2020). Um estudo de relato de caso descreveu o caso de um homem de 41 anos de idade, de Chengdu, na China, que, após ter se recuperado de COVID-19, foi reinternado com resultados positivos para esfregaços nasais, expectoração e fezes; no entanto, os resultados de RT-qPCR de esfregaços da garganta foram negativos (Li, Zang \& Zong, 2020).

Wang et al. relataram alguns casos identificados em Shenzhen, China, nos quais os resultados positivos recorrentes representaram 8,3\% (35 de 420) dos casos (Wang et al., 2020). Outro estudo conduzido em Xangai, China, relatou que 11 pacientes $(16,7 \%)$, cuja doença já se apresentava em estágio de convalescença, tiveram resultados positivos persistentes nas fezes para RNA viral (Ling et al., 2020).

Um relato de caso descreveu uma mulher de 72 anos da Coreia do Sul e destacou resultados positivos persistentes de RT-qPCR 6 dias após dois resultados negativos; embora ela tenha se recuperado completamente após ter realizado um segundo teste positivo (Chae et al., 2020). Outro estudo realizado na Coreia do Sul relatou que cinco indivíduos em 55 (9\%) tiveram reativação do SARS-CoV-2, dentre os quais quatro apresentavam sintomas leves e um era assintomático (Ye et al.2020).

Ravioli et al. conduziram um estudo na Suíça sobre a identificação de duas mulheres idosas. Elas tiveram resultados de teste positivos após 18 e 21 dias, respectivamente, de terem realizado dois exames com resultados negativos consecutivos para swabs nasofaríngeos (Ravioli, Ochsner \& Lindner, 2020).

Em abril de 2020, um relatório de caso da Coreia do Sul destacou que 163 de 7.829 pacientes $(2,1 \%)$ foram testados positivos após terem obtido melhora clínica (Kang \& Joo, 2020). Outro estudo realizado na Itália identificou um homem de 48 anos que desenvolveu uma forma grave de COVID-19. Ele se recuperou e teve alta após teste negativo com RT-qPCR, mas anticorpos IgM e IgG anti-SARS-CoV-2 foram detectados. Com o tempo, ele desenvolveu dispneia e dor no peito, e teve um reteste (Loconsole et al., 2020).

Dou et al. apresentaram um relato de Jiangsu, China, no qual um homem de 56 anos e sua filha (21 anos) foram diagnosticados com COVID-19 e receberam alta após terem seus resultados negativos. No entanto, 17 dias depois, ambos tiveram resultados positivos para os testes de RT-qPCR (Dou et al., 2020) Lan et al. em Wuhan, China, apresentaram um relatório no qual quatro profissionais médicos tiveram resultados de teste positivos após dois resultados negativos. Os testes de RT-qPCR foram repetidos 5 e 13 dias depois dos testes que haviam apresentado resultado negativo, e todos tiveram resultado positivo (Lan et al., 2020).

Zheng et al. relataram três casos de indivíduos que se recuperaram da COVID-19 e que receberam alta, uma semana depois; eles testaram positivo para swabs de nasofaringe e saliva durante o primeiro acompanhamento, ainda que com sintomas leves (Zheng et al., 2020). Um resumo dos relatórios anteriores é mostrado no Quadro 1, adaptado do trabalho de Osmaan, Daajani \& Alsahafi, 2020. 
Quadro 1. Resumo dos relatórios de casos de reinfecção do SARS-CoV-2.

\begin{tabular}{|c|c|c|c|c|c|}
\hline $\mathbf{N}^{\mathbf{0}}$ & Autoria & País/data & $\mathbf{N}$ & idade & $\begin{array}{l}\text { Simtomático/ } \\
\text { Assintomático }\end{array}$ \\
\hline 1 & KCDC, 2020 & Coréia do sul, maio 2020 & 285 & - & $126 / 158$ \\
\hline 2 & Zhang et al., 2020 & China, jan-fev 2020 & 7 & 10 meses-35 anos & $4 / 3$ \\
\hline 3 & Li et al., 2020 & China, fev 2020 & 19 & $18-71$ & $0 / 19$ \\
\hline 4 & Luo, 2020 & China, fev 2020 & 1 & 58 & $0 / 1$ \\
\hline 5 & Xing et al., 2020 & China, fev 2020 & 2 & 20,40 & $0 / 2$ \\
\hline 6 & Chen et al., 2020 & China, jan-fev 2020 & 4 & $12,29,38,49$ & $0 / 4$ \\
\hline 7 & Yuan et al., 2020 & China, jan-fev 2020 & 20 & $1-72$ & $0 / 20$ \\
\hline 8 & Li, Zang \& Zong 2020 & China, fev 2020 & 1 & 41 & $1 / 0$ \\
\hline 9 & Wang et al., 2020 & China, jan-mar 2020 & 35 & $21-45$ & $0 / 35$ \\
\hline 10 & Ling et al., 2020 & China, fev 2020 & 11 & $34-62$ & - \\
\hline 11 & Chae et al., 2020 & Coréia do Sul & 1 & 72 & - \\
\hline 12 & Ye et al., 2020 & China, fev 2020 & 5 & $27-42$ & $4 / 1$ \\
\hline 13 & Ravioli et al., 2020 & Suiça & 2 & 77,81 & $2 / 0$ \\
\hline 14 & Kang et al., 2020 & Coréia do Sul, abril 2020 & 163 & $1-35$ & $61 / 102$ \\
\hline 15 & Loconsole et al., 2020 & Itália, maio 2020 & 1 & 30 & $1 / 0$ \\
\hline 16 & Dou et al., 2020 & China, jan-fev 2020 & 2 & 17 & - \\
\hline 17 & Lan et al., 2020 & China, jan-fev 2020 & 4 & $5-13$ & $0 / 4$ \\
\hline 18 & Zheng et al., 2020 & China, jan-fev 2020 & 3 & 7 & $0 / 3$ \\
\hline
\end{tabular}

Fonte: Adaptado pelos autores conforme referência. Osmaan, Daajani \& Alsahafi (2020).

\subsection{A imunidade humoral e a reinfecção por SARS-CoV-2}

As imunidades humoral e mediada por células possuem papel importante para prevenir a reinfecção (Chandrashekar et al., 2020). O anticorpo contra SARS-CoV-2 pode ser encontrado na maioria dos pacientes com COVID-19 aproximadamente após 2 semanas de infecção (To et al., 2020), com uma produção de anticorpos neutralizantes mais rápida entre os casos graves (Liu et al., 2020).

Contudo, a diminuição dos níveis de anticorpos pode predispor os pacientes a reinfecções (Long et al., 2020). Alguns estudos de pacientes com COVID-19 mostraram que durante a infecção primária, a maioria dos anticorpos são nãoneutralizantes (Long et al., 2020), e o nível de anticorpos neutralizantes tende a ser baixo em casos leves, e altos em casos graves (Liu et al., 2020). 
No entanto, a maturação das células B após a primeira infecção pode resultar em células plasmáticas de vida longa e células B de memória. No decurso da reinfecção, essas células B de memória podem se diferenciar rapidamente em plasmoblastos, que por sua vez produzem anticorpos de alta afinidade mais rapidamente do que durante a infecção primária (To et al., 2020).

Dados do estudo realizado por To e colaboradores (2020) mostram que apesar da soroconversão de IgG, IgM não foi detectado por ensaio Imunofluorescência (IF) indireta até 8 dias após a segunda hospitalização. Esta falta de resposta de IgM é compatível com reinfecção. Além disso, os autores relatam que os resultados sugerem que o teste de IgM pode não ser útil para documentar infecção aguda para casos de reinfecção. Além disso, a IgM sérica não foi detectável por ensaio de anticorpo IF indireta usando linha celular infectada com SARS-CoV-2 no dia 10 após o início dos sintomas durante o primeiro episódio (To et al., 2020).

À medida que a titulação de anticorpos diminui no decorrer do tempo, a proporção de pacientes com COVID-19 que se torna suscetível a reinfecção cresce. Os estudos imunológicos desses casos de reinfecção podem possibilitar identificar os fatores que determinam o resultado da reinfecção. Identificar tais fatores torna-se especialmente importante visto que recentemente, um caso de reinfecção mostrou que a reinfecção pode evoluir de forma mais grave do que a primeira infecção (Wesiblum et al., 2020).

\section{Considerações Finais}

Embora os estudos sejam promissores para uma vacina eficaz nos próximos meses, a presença de mais de variantes genotípicas do vírus, a possibilidade de reinfecção e curta duração da soropositividade para anticorpos neutralizantes levantam a preocupação de que a vacinação pode resultar em uma imunidade pouco eficaz e de curto prazo contra SARS-CoV-2. Além disso, os níveis de imunoglobulina podem não se correlacionar com a eliminação viral e o risco de transmissibilidade de

\section{SARS-CoV-2.}

Além disso, a curta duração da imunidade contra o vírus pode não permitir o aumento da homogeneidade das populações afetadas em um período de tempo específico. Portanto, a imunidade coletiva pode não ser alcançada, visto que a reinfecção pode ocorrer mesmo na presença de anticorpos neutralizantes. Esses fatores aumentam a preocupação de que a eliminação da pandemia da COVID-19 pode não ser tão viável como antes assumido e que devem ser propagadas veementemente as medidas de prevenção da transmissão, enquanto mais aspectos do vírus e sua patogenicidade são investigados.

\section{Referências}

Centers for Disease Control and Prevention. (2020). Coronavirus disease 2019 (COVID-19) - symptoms. CDC. https:// www.cdc.gov/coronavirus/2019ncov/symptoms-testing/symptoms.html.

Chae, K. J., Jin, G. Y., Lee, C. S., Lee, H. B., Lee, J. H., \& Kwon, K. S. (2020). Positive con- version of COVID-19 after two consecutive negative RT-PCR results: a role of low-dose CT. European Journal of Radiology, 129:109122.

Chandrashekar, A., Liu, J., Martinot, A. J., McMahan, K., Mercado, N. B., Peter, L., Tostanoski, L. H., \& Barouch, D. H. (2020). SARS-CoV-2 infection protects against rechallenge in rhesus macaques. Science, 14(369), 812-817.

Chen, Y., Bai, W., Liu, B., Huang, J., Laurent, I., Chen, F., \& Xiao, X. (2020). Re-evaluation of retested nucleic acid-positive cases in recovered COVID-19 pa- tients: report from a designated transfer hospital in Chongqing, China. Journal of Infectology Public Health, 13(7): 932-934.

Dou, P., Zhang, S., Wang, C., Cai, L., Liu, Z., \& Xu, Q. (2020). Serial CT features in discharged COVID-19 patients with positive RT-PCR re-test. European Journal of Radiology, 127, 24-7.

Elias, C. S., Silva, L. A., Martins, M. T. S. L., Ramos, N. A. P., Souza, M. G. G., \& Hipólito, R. L. (2012). Quando chega o fim? Uma revisão narrativa sobre terminalidade do período escolar para alunos deficientes mentais. Revista Electrónica en Salud Mental, Alcohol y Drogas, 8(1), 48-53.

European Centre for Disease Prevention and Control. (2020). Novel coronavirus (SARS-CoV-2): discharge criteria for confirmed COVID-19 cases. ECDC Tech Rep, 1-5. 
Fiocruz. Primeiro caso de reinfecção pela Covid-19 no país é confirmado. <https://portal.fiocruz.br/noticia/primeiro-caso-de-reinfeccao-pela-covid-19-no-paise-confirmado>.

Hanaei, S., \& Rezaei, N. (2020). COVID-19: developing from an outbreak to a pandemic. Archives of Medical Research, 51(6):582-584.

Kang, Y-J., \& Joo, H. (2020). South Korea's COVID-19 infection status: from the perspective of reconfirmation after complete recovery. Journal of Pure and Applied Microbiology, 14, 1073-1075.

Kirkcaldy, R. D., King, B. A., \& Brooks, J. T. (2020). COVID-19 and postinfection immunity: limited evidence, many remaining questions. JAMA, 9;323(22):2245-2246.

Korea Centres for Disease Control and Prevention. (2020). Findings from investigation and analysis of re-positive cases. Press Release. News Room: KCDC. https://www.cdc.go.kr/board/ board.es?mid=a30402000000\&bid=0030\&act=view\&list_no=367267\&nPage=8. Acesso em 24/12/2020.

Lan, L., Xu, D., Ye, G., Xia, C., Wang, S., Li, Y., \& Xu, H. (2020). Positive RT-PCR test results in patients recovered from COVID-19. JAMA, 323(15), $1502-1503$.

Li, J., Wei, X., Tian, W., Zou, J., Wang, Y., Xue, W., \& Huang, W. (2020). Clinical features of discharged COVID-19 patients with an extended SARS-CoV-2 RNApositive signal in respiratory samples. Virus Research, 286:198047.

Li, X. J., Zhang, Z. W., \& Zong, Z. Y. (2020). A case of a readmitted patient who recovered from COVID-19 in Chengdu, China. Critical Care, 24: 8-10.

Ling, Y., Xu, S. B., Lin, Y. X., Tian, D., Zhu, Z. Q., Dai, F. H., \& Lu, H. Z. (2020). Persistence and clearance of viral RNA in 2019 novel coronavirus disease rehabilitation patients. Chinese Medical Journal (Engl), 133:1039-43.

Liu, L., To, K. K., Chan, K. H., Wong, Y. C., Zhou, R., Kwan, K. Y., \& Chen, Z. (2020). High neutralizing antibody titer in intensive care unit patients with COVID-19. Emerg Microbes Infections, 9(1), 1664-1670.

Loconsole, D., Passerini, F., Palmieri, V. O., Centrone, F., Sallustio, A., \& Pugliese, S. (2020). Recurrence of COVID-19 after recovery: a case report from Italy. Infection, 48, 965-967.

Lotfi, M., \& Rezaei, N. (2020). SARS-CoV-2: a comprehensive review from pathogenicity of the virus to clinical consequences. Journal of Medical Virology, 92:1864-1874.

Long, Q. X., Tang, X. J., Shi., Q. L., Li, Q., Deng, H. J., Yuan, J., \& Huang, A. (2020). Clinical and immunological assessment of asymptomatic SARS-CoV-2 infections. Nature Medicine, 26, 1200-1204.

Luo, A. Positive SARS-Cov-2 test in a woman with COVID-19 at 22 days after hospital discharge: a case report. Journal of Traditional Chinese Medical Sciences, 7(4): 413-417.

Osman, A. A., Al Daajani, M. M., Alsahafi, A. J. (2020). Re-positive coronavirus disease 2019 PCR test: could it be a reinfection? New Microbes New Infectology, 37,100748.

Randolph, H. E., \& Barriero, L. B. (2020). Herd immunity: understanding COVID-19. Immunity, 52(5):737-741.

Ravioli, S., Ochsner, H., \& Lindner, G. (2020). Reactivation of COVID-19 pneumonia: a report of two cases. Journal of Infectology, 12(3).

Roy, S. (2020). COVID-19 reinfection: myth or truth? SN Comprehensive Clinical Medicine, 2:710-713

Silva, W. M., Silva, M. E., Silva, W. B. de S., Santos, J. A., Gomes, M. C., Albuquerque, J. L. da S., Silva, G. C. dos S., Silva, E. R. B., e Silva, M. S., \& Silva, G. F. (2020). Caracterização das alterações cutâneas provocadas pelo novo Coronavírus SARS-CoV-2: uma revisão das novas evidências. Revista Eletrônica Acervo Saúde, 12(9), e4118.

Silva, M. S., Silva, W. M., Silva, G. M., Souza, R. G., Santos, J. A., Luz, M. K. S, Ferreira, M. D. F., Silva, T. M. L., Rocha, L. C. P., \& Silva, C. A. L. (2020). Manifestações neurológicas provocadas por COVID-19: uma revisão integrativa da literatura. Brazilian Journal of Development, 6(7), $52155-52163$.

To, K. K., Tsang, O, T., Leung, W. S., Tam, A. R., Wu, T.C., Lung, D.C., \& Yuen, k. (2020). Temporal profiles of viral load in posterior oropharyngeal saliva samples and serum antibody responses during infection by SARS-CoV-2: an observational cohort study. The Lancet Infectious Diseases, 20(5), 565-574.

Vosgerau, D. S. R., \& Romanowski, J. P. (2014). Estudos de revisão: implicações conceituais e metodológicas. Revista Diálogo Educacional, 14(41), 165189.

Wang, L. F., Yan, H., Zhang, D., Yun, Y., Zhang, C., An, C., \& Lei, L. (2020). Clinical characteristics and imaging findings of 35 patients recovered from COVID-19 with recurrent positive RT-PCR test results. SSRN Electron Journal. [preprint], https://doi.org/10.2139/ssrn.3564400.

Weisblum, Y., Schmidt, F., Zhang, F., DaSilva, J., Poston, D., Lorenzi, J. C. C., \& Bieniasz, P. D. (2020). Escape from neutralizing antibodies by SARS-CoV2 spike protein variants. BioRxiv, [preprint], 2020;10.1101/2020.07.21.214759.

World Health Organization. (2020). Coronavirus disease (COVID-19) pandemic. https://www.who.int/emergencies/diseases/ novel-coronavirus-2019.

Xing, Y., Mo, P., Xiao, Y., Zhao, O., Zhang, Y., \& Wang, F. (2020). Post-discharge surveillance and positive virus detection in two medical staff recovered from coronavirus disease 2019 (COVID-19), China, January to February 2020. Eurosurveillance, 25(10):pii=200019.

Yang, X., Yu, Y., Xu, J., Shu, H., Xia, J., Liu H., \& Shang, Y. (2020). Clinical course and outcomes of critically ill patients with SARS-CoV-2 pneumonia in Wuhan, China: a single-centered, retrospective, observational study. The Lancet Respiratory Medicine, 8:475-81. 
Research, Society and Development, v. 10, n. 1, e51910112016, 2021

(CC BY 4.0) | ISSN 2525-3409 | DOI: http://dx.doi.org/10.33448/rsd-v10i1.12016

Ye, G., Pan, Z., Pan, Y., Deng, Q., Chen, L., Li, J., \& Wang, X. (2020). Clinical characteristics of severe acute respiratory syndrome coronavirus 2 reactivation. Journal of Infect, 80:e14-7.

Yuan, B., Chen, Y., Zhang, K., \& Wang, C. (2020). Recurrence of positive SARS-CoV- 2 viral RNA in recovered COVID-19 patients during medical isolation observation. Nature Research, 10:1-12.

Zhang, B., Liu, S., Dong, Y., Zhang, L., Zhong, Q., \& Zou, Y., \& Zhang, S. (2020). Positive rectal swabs in young patients recovered from coronavirus disease 2019 (COVID-19). Journal of Infectology, 81(2): e49-e52.

Zheng, K. I., Wang, X. B., Jin, X. H., Liu, W. Y., Gao, F., Chen, Y. P., \& Zheng, M. (2020). A case series of recurrent viral RNA positivity in recovered COVID-19 Chinese patients. Journal of General Internal Medicine, 35(7), 2205-2206. 